\title{
EL FACTOR ECONÓMICO EN LA DESERCIÓN ESTUDIANTIL CASO: UNIVERSIDAD ANDINA DEL CUSCO, CICLOS 2017-I Y 2017-II
}

\author{
The economic factor in student desertion. Case: Universidad Andina del Cusco, cycles, 2017- \\ I and 2017-II
}

\author{
Corahua Ordóñez, Jéssika ${ }^{1,}$, Lozano Cusi, Rolando ${ }^{1}$
}

${ }^{1}$ Universidad Andina del Cusco, Cusco, Perú.

$\equiv$ jcorahua@uandina.edu.pe

\begin{abstract}
Resumen
La deserción universitaria es un problema que afrontan la mayoría de las universidades peruanas, según la agencia peruana de noticias 'Andina', la deserción universitaria en el Perú alcanza el 30\% y es motivada, especialmente, por la falta de una buena orientación vocacional y por razones económicas. Según el diario 'Gestión', el 27\% de ingresantes a universidades privadas abandonan su carrera en sus primeros años de estudios y, como uno de los factores principales son los problemas financieros. En términos económicos, la deserción representa una pérdida de al menos 100 millones de dólares; es por esta razón, el estudio de los factores de deserción estudiantil en la Universidad Andina del Cusco, ciclos 2017-I y 2017II; donde se evidencia que el factor económico predomina en la decisión del estudiantado para dejar sus estudios universitarios. La investigación tuvo un enfoque cuantitativo con diseño no experimental de corte transversal y de alcance descriptivo, que abarcó un universo de 337 estudiantes desertores, de un total de 1255 estudiantes no matriculados en los semestres de estudio (2017-I y 2017-II) en sus cinco facultades y 20 escuelas profesionales. La validez de constructo se realizó en una muestra piloto aplicado a 85 estudiantes a través del análisis factorial con rotación varimax $\mathrm{KMO}=0,698$ y Alfa de Crombach 0,786; el cuestionario estructurado se concretó con 21 reactivos considerando una escala Likert. El análisis estadístico refiere una descripción del factor económico detallado de modo comparativo por semestre académico en la sede central y 3 filiales, y también por facultades en la sede central de la Universidad Andina del Cusco.
\end{abstract}

Palabras clave: Factor económico, deserción.

\begin{abstract}
College desertion is a problem faced by most Peruvian universities, according to the Peruvian news agency 'Andina', university dropout in Peru reaches $30 \%$ and is motivated, especially by the lack of good vocational guidance and for economical reasons. According to the newspaper 'Gestión', 27\% of entrants to private universities abandon their careers in their first years of studies and, as one of the main factors, are the financial problems. In economic terms, the desertion represents a loss of at least 100 million dollars; it is for this reason, the study of the factors of student desertion in the Universidad Andina del Cusco, cycles 2017-I and 2017-II; evidencing that the economic factor predominates in the decision of the student body to leave their university studies. The research took a quantitative approach with non-experimental cross-sectional design and descriptive scope, which covered a universe of 337 drop-out students, out of a total of 1255 students not enrolled in the semesters of study (2017I and 2017-II) in its five faculties and 20 professional schools. The construct validity was performed in a pilot sample applied to 85 students through factor analysis with varimax rotation $\mathrm{KMO}=0.698$ and Crombach's Alpha 0.786; The structured questionnaire was completed with 21 items considering a Likert scale. The statistical analysis refers to a detailed description of the economic factor in a comparative way by academic semester at the headquarters and 3 subsidiaries and also by faculties at the headquarters of the Universidad Andina del Cusco.

Keywords: Economic factor, desertion.
\end{abstract}

Citar como: Corahua, J., Lozano, R. (2018). El factor económico en la deserción estudiantil caso: universidad andina del cusco, ciclos 2017-I y 2017-II, Rev Yachay, 7(1),347-354.

Recibido: 16-09-2018: Aceptado 07-12-2018

\section{Introducción}

La deserción estudiantil es uno de los problemas que abordan la mayoría de las instituciones de educación superior de toda Latinoamérica. La revista «Andina» (2017), cita a Justo Zaragoza, director de «Epouniversidad 2017», que refiere un estudio realizado por INSAN en 8 
universidades privadas de Lima, la cual señala que «la deserción universitaria en el Perú alcanza el 30\% y es motivada, especialmente, por la falta de buena orientación vocacional y por razones económicas.»

Según la Ley Universitaria 30220, promulgada en el 2014, establece directivas que regulan a todas las universidades públicas y privadas con fines de acreditación y certificación, donde la Universidad Andina del Cusco, no es ajena a estas disposiciones, tuvo que realizar cambios institucionales lo cual trajo consigo algunas disconformidades por parte de los estudiantes, reflejándose, en su mayoría, a los aspectos económicos ya que, además de efectuar pagos administrativos, gastos respecto a materiales de escritorio necesario en el desarrollo de las actividades académicas y, ahora, con la disposición de la SUNEDU por considerar 5 meses de estudio en el ciclo académico, lo que implica el pago de una mensualidad extra, generó para algunos estudiantes su no matrícula.

Escobar, V; Tirado, D; Santana, O. F.; Hernández, D.(2005), precisan que «deserción es el proceso de abandono voluntario o forzado de la carrera en la que se matricula un estudiante, por la influencia positiva o negativa de circunstancias internas o externas a él o ella», en lo que respecta a la educación superior entre uno y otro periodo académico (ciclo o año). Por otra parte: Rodríguez y Hernández (2008), indican que la deserción estudiantil se puede entender como la disolución del vínculo estipulado a través de la matrícula académica de manera biunívoca, ya sea por parte del estudiante o de la universidad, pero que en este proceso ambos se perjudican de modo financiero, académico y social. De modo similar Himmel (2017), refiere a la deserción universitaria como el abandono prematuro de un programa de estudios antes de alcanzar el título o grado y, considera un tiempo suficientemente largo para descartar la posibilidad de que el estudiante se reincorpore.

Diversas investigaciones realizadas respecto a la deserción estudiantil universitaria, están alineadas a enfoques que explicarían el porqué de la deserción estudiantil dentro de un campus universitario, según Quintero (2016), respecto al análisis que realiza sobre las causas de deserción universitaria, contempla 4 enfoques: el psicológico, el sociológico o ambiental, el económico, y el organizacional. Centrándonos en el enfoque económico, Quintero encuentra dos modelos:

a) Costo-beneficio: cuando los beneficios sociales y económicos generados por los estudios universitarios son percibidos como inferiores a los de los gastos universitarios, por lo que, el estudiante opta por retirarse debido a que percibe incapacidad para poder solventar los costos asociados a los estudios universitarios.

b) Focalización: los subsidios constituyen una forma de influir sobre la deserción cuando se dirigen a los grupos que presentan limitaciones reales para continuar sus estudios (Candamil, M; Parra, L; Sánchez, J., 2009).

Por otra parte, Sánchez, Navarro y García (2009) citando a Álvarez, J. M. (1997:49), asocia cuatro factores a las causas principales de la deserción estudiantil universitaria, que son los factores personales, factores académicos, factores institucionales y factores socio económicos; refiriendo que este último es generado por bajos ingresos familiares, desempleo, falta de apoyo familiar, incompatibilidad de horarios entre trabajo y estudio.

Por lo descrito, esta investigación tiene como objetivo determinar en qué medida el factor económico afecta la deserción universitaria de los estudiantes no matriculados en el ciclo académico 2017-I y 2017 -II de la Universidad Andina del Cusco, para que las autoridades universitarias tomen medidas convenientes a fin de que, la tasa de deserción no siga en aumento y, de esta manera, contribuya a que sus estudiantes concluyan sus estudios universitarios.

\section{Materiales y métodos}

La investigación presentó un enfoque cuantitativo. Este enfoque representa a un conjunto de procesos, es secuencial y probatorio. Cada etapa precede a la siguiente y no podemos brincar o eludir pasos (Canahuire Montufar, A.; Endara Mamani, F.; Morante Ríos, E., 2015), con un diseño no experimental, puesto que no existe la intención de modificar la realidad, y un alcance descriptivo a través de un análisis de medias.

La población objeto de estudio, estuvo conformado por 2685 alumnos no matriculados en el ciclo académico 2017-I y 2017-II, de la Universidad Andina del Cusco, considerando un muestreo probabilístico para una población finita, al 95\% de confiabilidad, proporción de éxito 50\%, proporción de fracaso 50\% y, error de estimación 5\%, se obtuvo el tamaño de muestra igual a 337 estudiantes que, de acuerdo a una estratificación proporcional por filiales en la Universidad Andina del Cusco, se tiene:

La recolección de datos se realizó a través de un cuestionario estructurado con 21 reactivos considerando una escala Likert, el resultado del análisis multivariado según el análisis factorial utilizando componentes principales y el método de varimax, resulta KMO $=0,698$ y prueba de Barlett sig $=0,000<0,05$, confirmando el uso del análisis factorial para la reducción de ítems propuestos en la investigación, con 4 componentes que explican la variable de estudio al 60,59\%. Por otro lado, la fiabilidad interna a través del alfa de 
Crombach que estima las correlaciones de los ítems, dio como resultado 0,786; confirmando que el cuestionario es fiable y proporciona resultados favorables para la investigación.

Tabla 1:

Muestra estratificada por filiales de la Universidad Andina del Cusco

\begin{tabular}{lcccccc}
\hline FILIALES & \multicolumn{2}{c}{ Ciclo 2017 - I } & Ciclo 2017 - II & Total & \\
& $\mathrm{N}_{1}$ & $\mathrm{n}_{1}$ & $\mathrm{~N}_{2}$ & $\mathrm{n}_{2}$ & $\mathrm{~N}_{\mathrm{T}}$ & $\mathrm{n}_{\mathrm{T}}$ \\
CUSCO & 863 & 108 & 991 & 124 & 1854 & 232 \\
PUERTO MALDONADO & 87 & 11 & 73 & 9 & 160 & 20 \\
QUILLABAMBA & 212 & 27 & 242 & 30 & 454 & 57 \\
SICUANI & 93 & 12 & 124 & 16 & 217 & 28 \\
Total & 1255 & 158 & 1430 & 179 & 2685 & 337 \\
\hline
\end{tabular}

Fuente: Servicios Académicos

\section{Resultados de la investigación}

Para determinar en qué medida el factor económico afecta la deserción universitaria en los estudiantes no matriculados en el ciclo académico 2017-I y 2017-II de la Universidad Andina del Cusco, se utilizó la tabla 2 que sirvió para las interpretaciones de las tablas y figuras estadísticas

Tabla 2

Tabla de baremación para medir 'Deserción universitaria'

\begin{tabular}{llll}
\hline Calificación & Nivel & Interpretación & Valoración \\
\hline \hline Nada/ No & $1-1,74$ & Afecta poco \\
Poco & $1,75-2,49$ & Afecta regularmente \\
Regular & $2,50-3,24$ & Afecta en gran medida \\
Bastante & $3,25-4$ & Afecta totalmente \\
\hline
\end{tabular}

1) Análisis del factor económico en la deserción universitaria por facultades en la sede central de la Universidad Andina del Cusco.

En la Facultad del CEAC, los componentes que afectan a la deserción universitaria en la Universidad Andina del Cusco, referido al factor económico en el ciclo 2017-I, en orden de prelación es: el costo de las mensualidades, la incompatibilidad horaria de sus actividades académicas con cualquier otra actividad, oportunidades laborales y, el incremento a 5 meses de estudio por ciclo, afecta en gran medida a la deserción en el ciclo 2017-II. El costo de las mensualidades y el incremento a 5 meses de estudio por ciclo afecta en gran medida a la deserción estudiantil universitaria.

Para la Facultad de Ciencias y Humanidades en el ciclo 2017-I, el costo de las mensualidades y el incremento a 5 meses de estudio por ciclo afecta totalmente a la deserción, en el ciclo 2017-II, la incompatibilidad horaria de sus actividades académicas con cualquier otra actividad, el costo de las mensualidades y el incremento a 5 meses de estudio por ciclo afecta en gran medida a la deserción estudiantil universitaria. 
Para la Facultad de Derecho y Ciencias Políticas en el ciclo 2017-I, el costo de las mensualidades afecta en gran medida a la deserción y, en el ciclo 2017-II la incompatibilidad horaria de sus actividades académicas con cualquier otra actividad afecta en gran medida a la deserción estudiantil universitaria.

Para la Facultad de Ingeniería y Arquitectura en el ciclo 2017-I, el incremento a 5 meses de estudio por ciclo afecta totalmente a la deserción, los gastos adicionales (trabajos, viajes, compra de materiales) y el exceso de trabajos (académicos, responsabilidad social, investigación) afecta en gran medida a la deserción y, en el ciclo 2017-II, el costo de las mensualidades en el aula afecta en gran medida a la deserción estudiantil universitaria.

Para la Facultad de Ciencias de la Salud en el ciclo 2017-I, el costo de las mensualidades afecta totalmente a la deserción, y en el ciclo 2017-II, el costo de las mensualidades y el incremento a 5 meses de estudio por ciclo afecta en gran medida a la deserción estudiantil universitaria (ver tabla 3).

Tabla 3

Descripción del factor económico en la deserción universitaria en los estudiantes no matriculados en el ciclo académico 2017 I y 2017-II de la Universidad Andina del Cusco por facultades, en la sede central.

\begin{tabular}{|c|c|c|c|c|c|c|}
\hline \multicolumn{2}{|l|}{ Factor Económico } & CEAC & $\begin{array}{c}\text { Cs y } \\
\text { Human. }\end{array}$ & $\begin{array}{c}\text { Derecho y Cs. } \\
\text { Políticas }\end{array}$ & $\begin{array}{l}\text { Ing. y } \\
\text { Arq. }\end{array}$ & $\begin{array}{c}\text { Cs. de la } \\
\text { Salud }\end{array}$ \\
\hline \multirow{2}{*}{$\begin{array}{l}\text { 1. Exceso de trabajos (académicos, } \\
\text { responsabilidad social, investigación) }\end{array}$} & 2017-I & 2,10 & 1,57 & 2,25 & 2,83 & 1,70 \\
\hline & 2017-II & 2,00 & 1,50 & 1,93 & 1,59 & 1,48 \\
\hline \multirow{2}{*}{$\begin{array}{l}\text { 2. El incremento a } 5 \text { meses de estudio por } \\
\text { ciclo }\end{array}$} & 2017-I & 2,54 & 3,57 & 3,00 & 3,27 & 2,95 \\
\hline & 2017-II & 2,65 & 2,00 & 2,93 & 2,34 & 2,52 \\
\hline \multirow{2}{*}{$\begin{array}{l}\text { 3. Gastos adicionales (trabajos, viajes, } \\
\text { compra de materiales) }\end{array}$} & 2017-I & 2,33 & 3,00 & 2,42 & 3,00 & 2,90 \\
\hline & 2017-II & 2,35 & 1,50 & 2,67 & 2,25 & 2,24 \\
\hline \multirow{2}{*}{ 4. El costo de las mensualidades } & 2017-I & 2,74 & 3,86 & 3,50 & 3,23 & 3,65 \\
\hline & 2017-II & 2,85 & 2,50 & 3,00 & 2,75 & 2,62 \\
\hline \multirow{2}{*}{$\begin{array}{l}\text { 5. Incompatibilidad horaria de sus } \\
\text { actividades académicas con cualquier otra } \\
\text { actividad }\end{array}$} & 2017-I & 2,74 & 2,57 & 3,25 & 2,87 & 2,80 \\
\hline & 2017-II & 2,46 & 3,00 & 3,27 & 2,22 & 1,90 \\
\hline \multirow{2}{*}{ 6. Oportunidades laborales } & 2017-I & 2,59 & 1,86 & 2,17 & 3,10 & 1,70 \\
\hline & 2017-II & 2,39 & 2,10 & 2,67 & 2,09 & 1,90 \\
\hline
\end{tabular}

Fuente: Cuestionario aplicado a estudiantes no matriculados de la UAC-2017

\begin{tabular}{|l|l|l|l|l|}
\hline Afecta & Poco $(1-1,74)$ & Regular $(1,75-2,49)$ & En gran medida $(2,50-3,24)$ & Totalmente $(3,25-4)$ \\
\hline
\end{tabular}

El factor económico en el ciclo 2017-I afectó en gran medida a la deserción estudiantil evidenciándose mayormente en la Facultad de Ingeniería y Arquitectura, seguido por Derecho y Ciencias Políticas y, para el ciclo 2017-II, el factor económico afectó en gran medida 
en la Facultad de Derecho y Ciencias Políticas y, regularmente en las facultades de CEAC y Ciencias y Humanidades (ver Tabla 4, figura 1).

Tabla 4

Efecto del factor económico en la deserción universitaria en los estudiantes no matriculados en el ciclo académico 2017-I y 2017-II de la Universidad Andina del Cusco, por facultades en la sede central.

\begin{tabular}{lcc}
\hline Facultades & Ciclo 2017-I & Ciclo 2017-II \\
\hline \hline CEAC & 2,51 & 2,45 \\
Ciencias y Humanidades & 2,74 & 2,10 \\
Derecho y Ciencias Políticas & 2,76 & 2,74 \\
Ingeniería y Arquitectura & 3,05 & 2,21 \\
Ciencias de la Salud & 2,62 & 2,11 \\
\hline Total & 2,72 & 2,34 \\
\hline
\end{tabular}

Fuente: Cuestionario aplicado a estudiantes no matriculados de la UAC - 2017

\begin{tabular}{|l|l|l|l|l|}
\hline Afecta & Poco $(1-1,74)$ & Regular $(1,75-2,49)$ & En gran medida $(2,50-3,24)$ & Totalmente $(3,25-4)$ \\
\hline
\end{tabular}

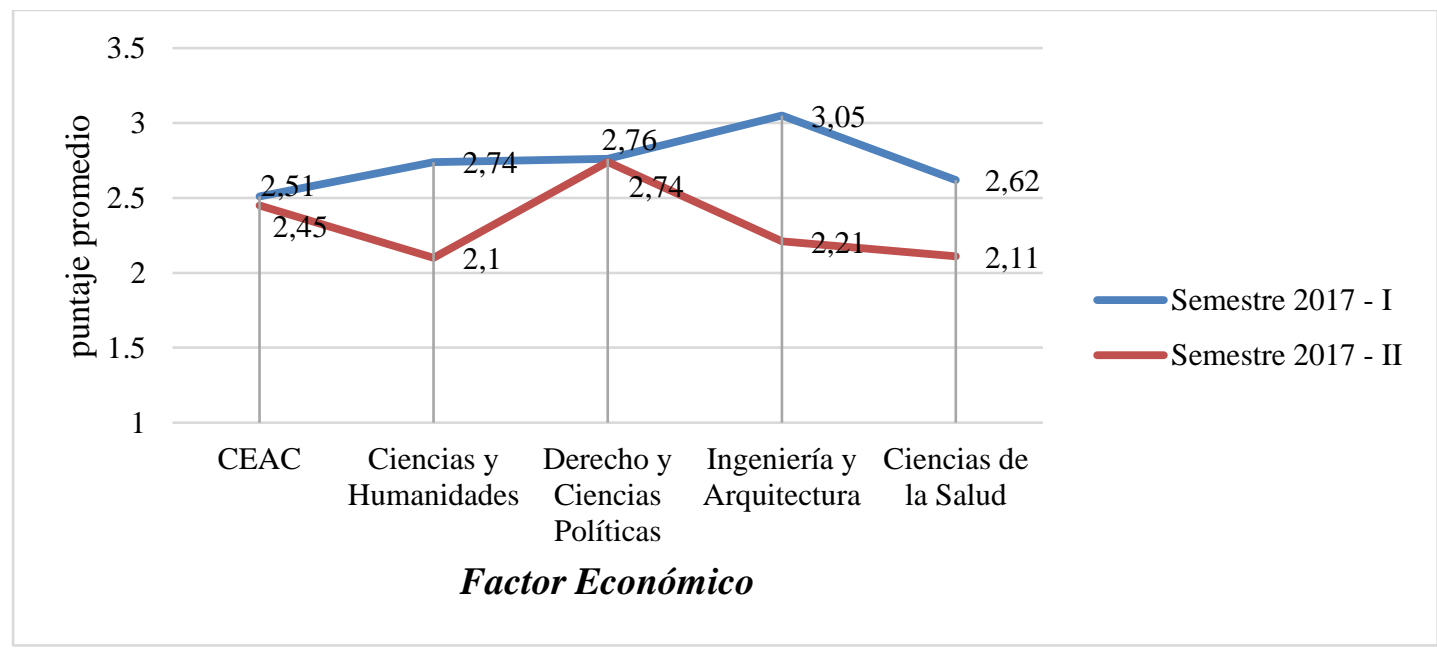

Fuente: Cuestionario aplicado a estudiantes no matriculados de la UAC - 2017

Figura 1. Efecto del factor económico en la deserción universitaria en los estudiantes no matriculados en el ciclo académico 2017-I y 2017-II de la Universidad Andina del Cusco, por facultades en la filial central.

2) Análisis del factor económico en la deserción universitaria por filiales de la Universidad Andina del Cusco.

Entre los componentes que afectan a la deserción universitaria en la filial central de la Universidad Andina del Cusco, referido a aspectos económicos para el ciclo académico 2017-I, están: el costo de las mensualidades que afecta en gran medida, seguido por el incremento a 5 meses de estudio por ciclo, el cual también se sigue evidenciado para el ciclo 2017-II. 
Para la filial de Puerto Maldonado, se evidencia que la incompatibilidad horaria de sus actividades académicas con otra actividad afecta totalmente a la deserción estudiantil en el ciclo 2017-I; para el ciclo 2017-II afectaba en gran medida a la deserción el costo de las mensualidades.

Para la filial de Quillabamba se evidencia que la incompatibilidad horaria de sus actividades académicas con otra actividad y el costo de las mensualidades afecta, en gran medida, a la deserción estudiantil para el ciclo 2017-I. Para el ciclo 2017-II el incremento a 5 meses de estudio por ciclo afectaba en gran medida a la deserción estudiantil.

Para la filial Sicuani, se evidencia que la incompatibilidad horaria de sus actividades académicas con otra actividad y el costo de las mensualidades afecta en gran medida a la deserción estudiantil para el ciclo 2017-I; para el ciclo 2017-II el incremento a 5 meses de estudio por ciclo afectaba en gran medida a la deserción estudiantil. (Ver tabla 5).

Tabla 5

Descripción por filiales del factor económico en la deserción universitaria en los estudiantes no matriculados en el ciclo académico 2017-I y 2017-II de la Universidad Andina del Cusco.

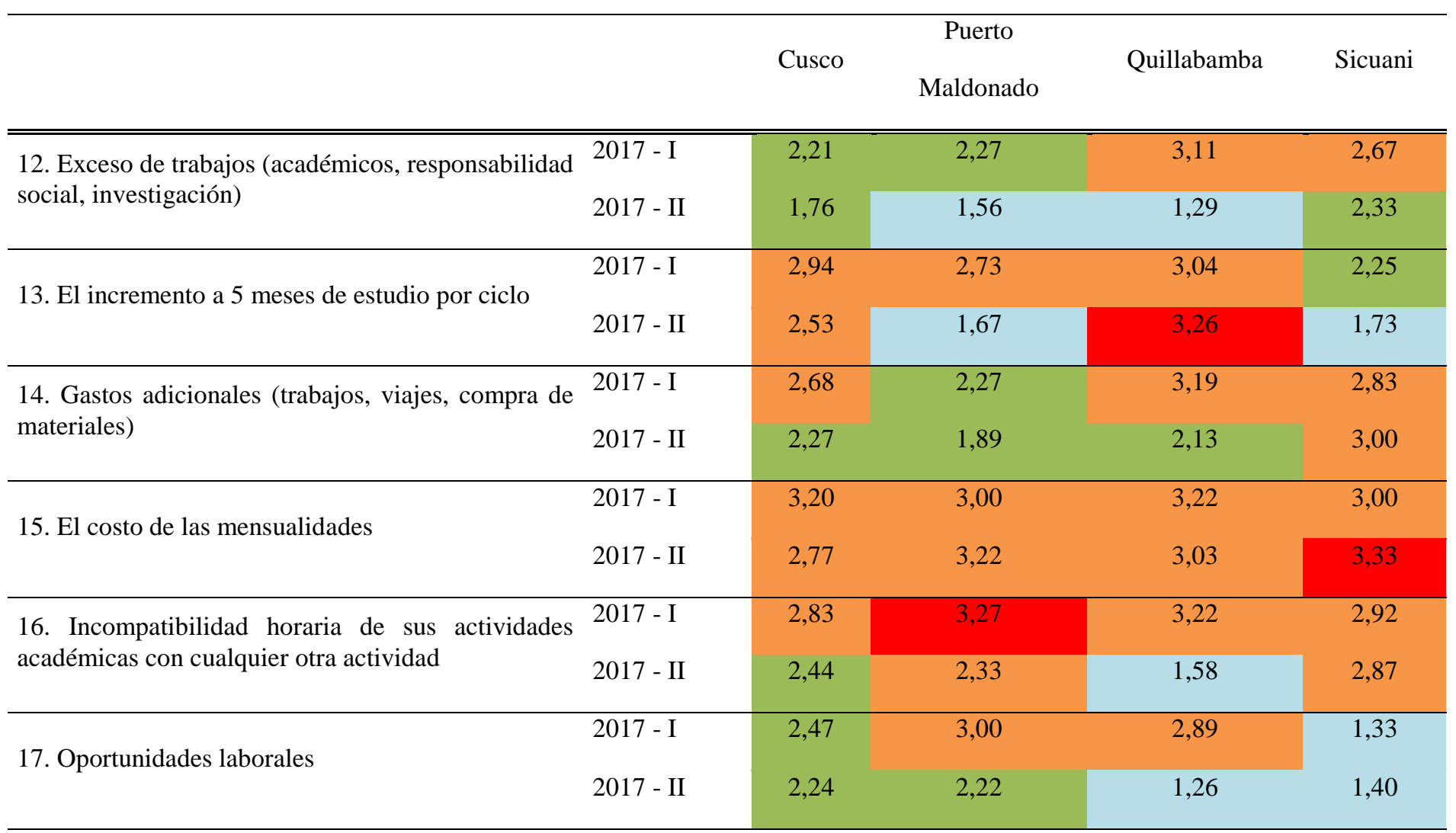

Fuente: Cuestionario aplicado a estudiantes no matriculados de la UAC - 2017

\begin{tabular}{|l|l|l|l|l|}
\hline Afecta & Poco $(1-1,74)$ & Regular $(1,75-2,49)$ & En gran medida $(2,50-3,24)$ & Totalmente $(3,25-4)$ \\
\hline
\end{tabular}

En todas las filiales de la Universidad Andina del Cusco, en el Ciclo 2017-I, se observa cómo el 'factor económico' afectó en gran medida a la deserción universitaria, mucho más en la filial de Quillabamba, seguido por Puerto Maldonado; para el ciclo 2017-II este factor afectó regularmente a la deserción; más aún, en la filial de Sicuani, seguido por la sede central del Cusco (ver tabla 6, figura 2). 
Tabla 6

Efecto del factor económico en la deserción universitaria en los estudiantes no matriculados en el ciclo académico 2017-I y II de la Universidad Andina del Cusco, por filial.

\begin{tabular}{lcc}
\hline FILIAL & Ciclo 2017-I & Ciclo 2017-II \\
\hline \hline Cusco & 2,72 & 2,34 \\
Puerto Maldonado & 2,76 & 2,15 \\
Quillabamba & 3,11 & 2,09 \\
Sicuani & 2,50 & 2,44 \\
\hline Total & 2,77 & 2,29 \\
\hline
\end{tabular}

Fuente: Cuestionario aplicado a estudiantes no matriculados de la UAC-2017

\begin{tabular}{|l|l|l|l|l|}
\hline Afecta & Poco $(1-1,74)$ & Regular $(1,75-2,49)$ & En gran medida $(2,50-3,24)$ & Totalmente $(3,25-4)$ \\
\hline
\end{tabular}

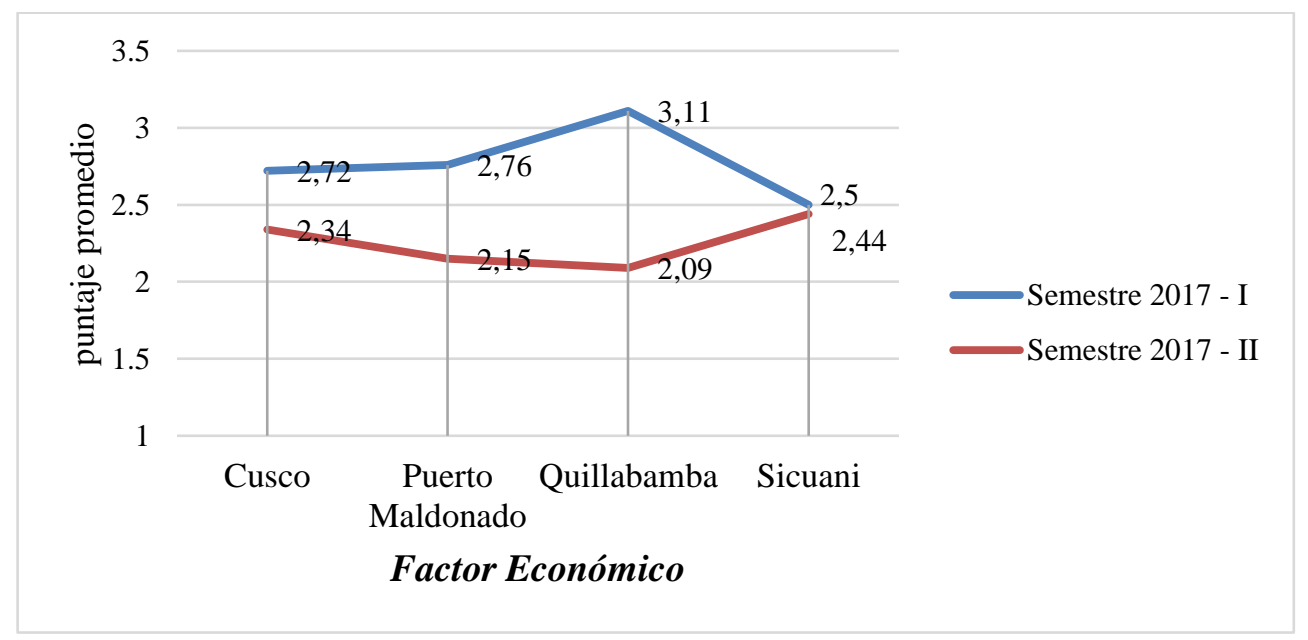

Fuente: Cuestionario aplicado a estudiantes no matriculados de la UAC - 2017

Figura 2. Efecto del Factor Económico en la Deserción Universitaria en los estudiantes no matriculados en el ciclo académico 2017-I y II de la Universidad Andina del Cusco, por filial.

\section{Discusión}

El factor económico coadyuvó en gran medida a la deserción universitaria de los alumnos de pregrado de la Universidad Andina del Cusco, en los ciclos académicos 2017-I y 2017-II, debido al costo de las mensualidades, la incompatibilidad horaria de sus actividades académicas con otra actividad usualmente porque trabajan y, el incremento a 5 meses de estudio por ciclo. En este sentido, se pudo observar que la situación de los estudiantes se ve afectada, puesto que en muchas ocasiones deben de trabajar para sustentar el pago de sus mensualidades, descuidando sus estudios. Rodríguez y Hernández (2017), aclaran muy bien que el factor que predomina en la deserción es la pobreza que obliga a los jóvenes a buscar un empleo. 


\section{Conclusiones}

El factor económico en el ciclo académico 2017-I, contribuyó en gran medida a la deserción estudiantil en la Universidad Andina del Cusco, mucho más a la filial de Quilllabamba, donde el costo de las mensualidades y la incompatibilidad horaria de sus actividades académicas con otras actividades — como trabajar — contribuye en gran medida a la deserción estudiantil en este ciclo académico.

Haciendo un análisis en la sede central de Cusco, el factor económico en el ciclo académico 2017-I, contribuyó en gran medida para la deserción estudiantil en todas las facultades. Sobre todo, afectó a la Facultad de Ingeniería y Arquitectura, donde el incremento de 4 a 5 meses de estudio por ciclo, contribuyó totalmente con la deserción de los estudiantes de esta facultad, además del costo de las mensualidades, las oportunidades laborales que se le presentaron y los gastos adicionales, como compra de materiales, son indicadores que contribuyen con la deserción estudiantil. De la misma manera, la Facultad de Derecho y Ciencias Políticas se vio afectada debido al costo de las mensualidades y la incompatibilidad horaria de sus actividades académicas con el trabajo que contribuyó totalmente a la deserción en esta facultad.

En el ciclo académico 2017-II, el factor económico, contribuyó regularmente a la deserción estudiantil de modo general, sin embargo, en la filial de Sicuani, el costo de las mensualidades contribuyó totalmente a la deserción estudiantil. Para la filial de Quillabamba, el incremento a 5 meses de estudio por ciclo, contribuyó totalmente con la deserción.

Por otra parte, en la sede central del Cusco en el ciclo académico 2017-I, la Facultad de Derecho y Ciencias Políticas se vio afectada, en gran medida, por la deserción de sus estudiantes debido al factor económico y, en regular medida, en las facultades de CEAC y Ciencias y Humanidades; donde el costo de las mensualidades y la incompatibilidad horaria académica contribuye totalmente con la deserción de los estudiantes de la Facultad de Derecho; para la Facultad de Ciencias y Humanidades, también la incompatibilidad horaria de sus actividades académicas y el costo de las mensualidades contribuyen en gran medida a la deserción estudiantil en la Universidad Andina del Cusco.

\section{Referencias bibliográficas}

Canahuire Montufar, A.; Endara Mamani, F.; Morante Ríos, E. (2015). ¿Cómo hacer la tesis universitaria? Una guía para investigadores. Cusco, Perú: Colorgraf.

Candamil, M; Parra, L; Sánchez, J. (2009). http://docplayer.es/44447322-Analisis-de-la-desercionestudiantil-en-la-universidad-de-caldas.html. Recuperado el 5 de noviembre de 2018, de http://sig.ucaldas.edu.co/gestionDocumental/SII/anàlisis_ indicadores/soporte_acredita_13

Escobar, V; Tirado, D; Santana, O. F.; Hernández, D. (2005). Estudio sobre la deserción y repitencia en la educación superior en Panamá. Consejo de Rectores de Panamá . Panamá: Consejo de Rectores. Recuperado el 4 de noviembre de 2018, de http://www.alfaguia.org/alfaguiav2/files/1342817723_34 30.pdf

Hernández Sampieri, R., Fernández Collado , C., \& Baptista Lucio, M. (2014). Metodologia de la Investigación (Sexta ed.). Mexico D.F: Mc Graw Hill.

Himmel, E. (2 de 9 de 2017). Modelo de análisis de la deserción estudiantil en la educación superior. Obtenido de http://www.cse.cl/doc/web.csepublic_2100_Himmel2200 2.pdf

Noticias, A. P. (Junio de 2017). Andina. Obtenido de https://andina.pe/agencia/noticia-educacion-universitaria- hay-30-desercion-falta-orientacion-y-recursos670189.aspx

Quintero Velasco, I. (2016). Repositorio de la Universidad Nacional Abierta y a Distancia UNAD. Recuperado el noviembre5 de 2018, de https://repository.unad.edu.co/bitstream/10596/6253/1/23 783211.pdf

Rodriguez, J., \& Hernandez, J. (5 de 9 de 2017). Redalyc. Obtenido de htpp://redalyc.uaemex.mx/pdf/447/44780116.pdf

Rodriguez, J; Hernández, J. (2008). La deserción universitaria en México. La experiencia de la Universidad Autónoma Metropolitana Campus Iztapalapa. Instituto de investigación en educación. Actualidades investigativas en educación, $8(1), \quad 1$ - $31 . \quad$ Obtenido de http://redalycuaemex.mx/pdf/447/44780116.pdf

Sánchez Amaya, G., Navarro Salcedo, W., \& García Valencia, A. (Mayo de 2009). Factores de deserción estudiantil en la universidad Surcolombiana. Facultad de educación. Universidad Surcolombiana. Obtenido de http://www.alfaguia.org/alfaguia/files/1319757570_14.pd f

Valderrama Pérez, Y., \& Paúcar Ccahuana, R. (09 de Octubre de 2016). Repositorio Unsaac. Recuperado el 27 de Agosto de 2018 http://repositorio.unsaac.edu.pe/handle/UNSAAC/120 de 